\title{
Algebraic approach for shape invariant potentials in Klein-Gordon equation
}

\author{
${ }^{a}$ M. R. Setare ${ }^{1 b}$ O. Hatami ${ }^{2}$ \\ ${ }^{a} 1$ Department of Science, Payame Noor University, Bijar, Iran \\ ${ }^{b}$ Department of Science, Payame Noor University, Sarpolzahab, Iran
}

\begin{abstract}
The Shape invariant method has the algebraic structure and its algebras are infinite-dimensional. These algebras are converted into finite-dimensional under conditions. Based on the property of this method we obtain the algebraic structure of some physical potentials in the s-wave Klein-Gordon equation with scslar and vector potential which satisfy the Shape invariant condition.
\end{abstract}

keywords: Klein-Gordon equation; Shape Invariance; Lie algebra.

PACS number : 03.65.Fd, 03.65.Ge, 02.20.Sv

\footnotetext{
${ }^{1}$ E-mail: rezakord@ipm.ir

${ }^{2}$ E-mail:hatami_physics@yahoo.com
} 


\section{Introduction}

In recent years there has been an increasing interest in the study of relativistic wave equations, particularly, the Klein-Gordon equation [1, 2, 3, 4], because the solutions of this equation play a fundamental role in describing the relativistic effects in nuclear physics and high energy physics, and other areas.

On the other hand, the supersymmetry quantum mechanics and shape invariant method have an underly algebraic structure and the associated Lie algebras are shown to be infinite dimensional [5]. This method is an exact and elegant technique for determining the eigenvalues and eigenfunctions of quantum mechanical problems. Supersymmetry was first studied in the simplest case of supersymmetric quantum mechanics by Witten [6], Cooper and Freeman [7]. In 1983, the concept of shape invariant potential was introduced by Gendenstein [8], there has been considerable discussion in the literature of this technique and it has been applied to many of the solvable systems in quantum mechanics $[7,9]$. It is well known that a potential is said to be shape invariant, if its supersymmetry partner potential has the same spatial dependence as the original potential with possibly altered parameters, and the corresponding Hamiltonian can be solved [2, 3, 7, 9, 10, 11].

In this Letter we utilize the approach of Balantekin [5] for shape invariant potentials. In section 2 of this paper we provide a brief review of the shape invariance method and associated Lie algebra. In section 3 we represent the Klein-Gordon equation with equal scalar and vector five-parameter exponential-type potentials, then we show a large class of exponential-type potentials can be obtain by choosing the appropriate parameters in this potential. We show that the Lie algebra of fiveparameter exponential-type potentials is $s u(1,1)$, then in the same process we determine the Lie algebra structure of Harmonic Oscillator with Heisenberg-Wely algebra, and Morse potential with $s u(1,1)$ algebra in the Klein-Gordon equation. Finally, the conclusion is given in section 4 .

\section{Supersymmetry quantum mechanics and algebraic formulation to shape invariance}

We note that supersymmetry method is based on the relation among the energy spectrum, energy eigenfunction and phase shift of the Hamiltonians associated with two supersymmetric partner potentials $V_{-}(x)$ and $V_{+}(x)$ [7]. Let us assume that the ground state energy being zero, namely,

$$
H_{-}(x)=H(x)-E_{0},
$$

where $E_{0}$ is the ground state energy of $H(x)$, and introduce the following operators:

$$
\hat{A}=\frac{d}{d x}+W(x), \quad \hat{A}^{\dagger}=-\frac{d}{d x}+W(x),
$$


where $W(x)$ signifies the superpotential. From these two operators we can construct two partner Hamiltonians

$$
\begin{aligned}
& \hat{H}_{-}=\hat{A^{\dagger}} \hat{A}=-\frac{d^{2}}{d x^{2}}+V_{-}(x), \\
& \hat{H}_{+}=\hat{A} \hat{A}^{\dagger}=-\frac{d^{2}}{d x^{2}}+V_{+}(x) .
\end{aligned}
$$

Since $\hat{A}$ annihilates the ground state

$$
\hat{A} \psi_{0}(x)=\left(\frac{d}{d x}+W(x)\right) \psi_{0}(x)=0 .
$$

The ground state wave function is obtained as:

$$
\psi_{0}(x) \sim \exp \left(-\int_{x_{0}}^{x} W(x) d x\right)
$$

where $x_{0}$ is an arbitrarily chosen reference point. A supersymmetric pair of potentials

$$
V_{ \pm}\left(x ; a_{1}\right)=W^{2}\left(x ; a_{1}\right) \pm W^{\prime}\left(x ; a_{1}\right)
$$

are called shape invariant if

$$
V_{+}\left(x ; a_{1}\right)=V_{-}\left(x ; a_{2}\right)+R\left(a_{1}\right),
$$

meaning that two supersymmetric partner potentials have the same form, but are characterized by different values of parameters $a_{1}$ and $a_{2}$. The remainder $R\left(a_{1}\right)$ is independent of $x$ and the parameter $a_{2}$ is a function of the parameter $a_{1}$. So far two classes of shape invariant are studied and discussed, in one class the parameters are related to each other by a translation $[12,13]$

$$
a_{2}=a_{1}+\eta
$$

and in second class they are related by a scaling $[14,15]$

$$
a_{2}=q a_{1}
$$

In this paper we consider the translation case. We define an operator which transforms the parameters of partner potential [12]

$$
\hat{T}\left(a_{1}\right) O\left(a_{1}\right) \hat{T}^{-1}\left(a_{1}\right)=O\left(a_{2}\right),
$$

where $\hat{T}\left(a_{1}\right)$ and $\hat{T}^{-1}\left(a_{1}\right)$ is as follows

$$
\hat{T}\left(a_{1}\right)=\exp \left(\eta \frac{\partial}{\partial \mathrm{a}_{1}}\right), \quad \hat{\mathrm{T}}^{-1}\left(\mathrm{a}_{1}\right)=\hat{\mathrm{T}}^{\dagger}\left(\mathrm{a}_{1}\right)=\exp \left(-\eta \frac{\partial}{\partial \mathrm{a}_{1}}\right) .
$$

We introduce new operators in the following form to establish the algebraic structure

$$
\hat{B}_{+}=\hat{A}^{\dagger}\left(a_{1}\right) \hat{T}\left(a_{1}\right)
$$




$$
\hat{B}_{-}=\hat{B}_{+}^{\dagger}=\hat{T}^{\dagger}\left(a_{1}\right) \hat{A}\left(a_{1}\right)
$$

So the Hamiltonians of Eqs. (3), (4) can be written as

$$
\hat{H}_{-}=\hat{A}^{\dagger} \hat{A}=\hat{B}_{+} \hat{B}_{-}, \quad \hat{H}_{+}=\hat{A} \hat{A}^{\dagger}=\hat{T} \hat{B}_{-} \hat{B}_{+} \hat{T}^{\dagger} .
$$

Eq. (8) can be written as a commutator

$$
\left[\hat{B}_{-}, \hat{B}_{+}\right]=\hat{T}^{\dagger}\left(a_{1}\right) R\left(a_{1}\right) \hat{T}\left(a_{1}\right) \equiv R\left(a_{0}\right)
$$

where we defined

$$
a_{n}=a_{1}+(n-1) \eta
$$

and we have used

$$
R\left(a_{n}\right)=\hat{T}\left(a_{1}\right) R\left(a_{n-1}\right) \hat{T}^{\dagger}\left(a_{1}\right),
$$

valid for any $n$. We defined the commutation relation (16) for determine the Lie algebra associated by the shape-invariant and also this equation shows that $\hat{B}_{-}$and $\hat{B}_{+}$are the appropriate creation and annihilation operators for the spectra of the shape-invariant potentials provided that their noncommutativity with $R\left(a_{0}\right)$ is taken into account. The additional relations

$$
\begin{aligned}
& R\left(a_{n}\right) \hat{B}_{+}=\hat{B}_{+} R\left(a_{n-1}\right), \\
& R\left(a_{n}\right) \hat{B}_{-}=\hat{B}_{-} R\left(a_{n+1}\right)
\end{aligned}
$$

which readily follow from Eqs. (13), (14), (18). One can show the following relations

$$
\begin{aligned}
& \hat{B}_{-}\left|\psi_{0}\right\rangle=\hat{A}\left|\psi_{0}\right\rangle=0, \\
& {\left[\hat{H}, \hat{B}_{+}^{n}\right]=\left(R\left(a_{1}\right)+R\left(a_{2}\right)++R\left(a_{n}\right)\right) \hat{B}_{+}^{n},} \\
& {\left[\hat{H}, \hat{B}_{-}^{n}\right]=-\hat{B}_{-}^{n}\left(R\left(a_{1}\right)+R\left(a_{2}\right)+. .+R\left(a_{n}\right)\right),}
\end{aligned}
$$

meaning that $\hat{B}_{+}^{n}\left|\psi_{0}\right\rangle$ is an eigenstate of the Hamiltonian with the eigenvalue $R\left(a_{1}\right)+R\left(a_{2}\right)+. .+R\left(a_{n}\right)$. The normalized wavefunction is

$$
\left|\psi_{n}\right\rangle=\frac{1}{\sqrt{R\left(a_{1}\right)+R\left(a_{2}\right)++R\left(a_{n}\right)}} \hat{B}_{+} \cdot \cdot \frac{1}{\sqrt{R\left(a_{1}\right)+R\left(a_{2}\right)}} \hat{B}_{+} \frac{1}{\sqrt{R\left(a_{1}\right)}} \hat{B}_{+}\left|\psi_{0}\right\rangle
$$

where $\left|\psi_{0}\right\rangle$ depends on $x$ and parameter $a_{1}$. It is easily to show the commutation relations

$$
\begin{gathered}
{\left[\hat{B}_{+}, R\left(a_{0}\right)\right]=\left(R\left(a_{1}\right)-R\left(a_{0}\right)\right) \hat{B}_{+},} \\
{\left[\hat{B}_{+},\left(R\left(a_{1}\right)-R\left(a_{0}\right)\right) \hat{B}_{+}\right]=\left(R\left(a_{2}\right)-2 R\left(a_{1}\right)+R\left(a_{0}\right)\right) \hat{B}_{+}^{2},}
\end{gathered}
$$

and so on. There is an infinite number of such commutation relations. If $R\left(a_{n}\right)$ satisfy certain relations one of the commutators in this series may vanish. These commutation relations with their 
complex conjugates form a Lie algebra with a finite number of elements. We know that for confining potentials the $n$th eigenvalue for large $n$ obeys the constraint [16]

$$
E_{n} \leq \text { constant } \times n^{2}
$$

We consider some potentials where have a finite algebra, for these potentials $E_{n}$ is as

$$
E_{n}=\mu n^{2}+\nu n+\kappa,
$$

where $\mu, \nu, \kappa$ are constants. By using Eq. (28) one can obtain

$$
R\left(a_{n}\right)=E_{n}-E_{n-1}=2 \mu n+\nu-\mu
$$

then we have

$$
R\left(a_{n}\right)-R\left(a_{n-1}\right)=2 \mu
$$

So, the Lie algebra is finite

$$
\left[\hat{B}_{-}, \hat{B}_{+}\right]=R\left(a_{0}\right), \quad\left[\hat{B}_{-}, R\left(a_{0}\right)\right]=-2 \mu \hat{B}_{-}, \quad\left[\hat{B}_{+}, R\left(a_{0}\right)\right]=2 \mu \hat{B}_{+} .
$$

For the case $\mu=0$ it is Heisenberg-Wely algebra, if $\mu \neq 0$ the algebra is either $S U(1,1)$ or $S U(2)$ depends on the sign of $\mu$. The energy spectrum corresponding to $H(x)$ can be derived from the shape-invariance conditions

$$
E_{n}=\sum_{k=1}^{n} R\left(a_{k}\right)+E_{0}
$$

\section{EXAPLES}

Now we would like to apply the discussion in the previous section for some potentials which satisfy the shape invariant condition, then we determine the Lie algebra of these potentials.

\subsection{Five-parameter exponential-type potential model}

The s-wave Klein-Gordon equation with scalar and vector potentials is $(\hbar=c=1)[1]$

$$
\left\{\frac{d^{2}}{d r^{2}}+[E-V(r)]^{2}-[M+S(r)]^{2}\right\} u(r)=0,
$$

where the radial wave function is $R(r)=u(r) / r$ and $M$ is the mass, $E$ is the energy and $S(r)$ and $V(r)$ denote the scalar and vector potentials, respectively. If $S(r) \geq V(r)$, there exists bound state solution. In the case of equal scalar and vector potentials, $S(r)=V(r)$, Eq. (1) reduces to a Schrödinger-like equation

$$
\left\{-\frac{d^{2}}{d r^{2}}+2(M+E) V(r)\right\} u(r)=\left(E^{2}-M^{2}\right) u(r)
$$


We consider the five-parameter exponential-type potential model, which is written in the form of [17]

$$
V(r)=\frac{Q_{3}^{2}+\mathrm{g}-\frac{\mathrm{Q}_{2}^{2}}{\mathrm{q}}+2 \alpha \mathrm{Q}_{2}}{e^{2 \alpha r}+q}+\frac{Q_{2}^{2}-q Q_{3}^{2}-2 \alpha q Q_{2}}{\left(e^{2 \alpha r}+q\right)^{2}}+\frac{\mathrm{g} \frac{\mathrm{Q}_{3}}{\mathrm{Q}_{2}}-\frac{\mathrm{Q}_{2} \mathrm{Q}_{3}}{\mathrm{q}}+\alpha \mathrm{Q}_{3}}{e^{2 \alpha r}+q} e^{\alpha r}+\frac{2 Q_{2} Q_{3}-2 \alpha q Q_{3}}{\left(e^{2 \alpha r}+q\right)^{2}} e^{\alpha r},
$$

where the range of parameter $q$ is $-1 \leq q<0$ or $q>0$. Combining Eqs. (34), (35) leads to

$$
\begin{aligned}
\left\{-\frac{d^{2}}{d r^{2}}+2(m+E)\{\right. & \frac{Q_{3}^{2}+\mathrm{g}-\frac{\mathrm{Q}_{2}^{2}}{\mathrm{q}}+2 \alpha \mathrm{Q}_{2}}{e^{2 \alpha r}+q}+\frac{Q_{2}^{2}-q Q_{3}^{2}-2 \alpha q Q_{2}}{\left(e^{2 \alpha r}+q\right)^{2}} \\
& \left.+\frac{\mathrm{g} \frac{\mathrm{Q}_{3}}{\mathrm{Q}_{2}}-\frac{\mathrm{Q}_{2} \mathrm{Q}_{3}}{\mathrm{q}}+\alpha \mathrm{Q}_{3}}{e^{2 \alpha r}+q} e^{\alpha r}+\frac{2 Q_{2} Q_{3}-2 \alpha q Q_{3}}{\left(e^{2 \alpha r}+q\right)^{2}} e^{\alpha r}\right\} u(r)=\left(E^{2}-m^{2}\right) u(r) .
\end{aligned}
$$

The corresponding superpotential is

$$
W\left(r, a_{n}\right)=\frac{\mathrm{g}}{2 a_{n}}-\frac{a_{n}}{2 q}+\frac{a_{n}}{e^{2 \alpha r}+q}+\frac{Q_{3}}{e^{2 \alpha r}+q} e^{\alpha r},
$$

where

$$
a_{n}=Q_{2}+2 n \alpha q
$$

The remainder in Eq. (8) is given by

$$
R\left(a_{n}\right)=-\left[\frac{\mathrm{g}}{2\left(Q_{2}+2 n \alpha q\right)}-\frac{\left(Q_{2}+2 n \alpha q\right)}{2 q}\right]^{2}+\left[\frac{\mathrm{g}}{2\left(Q_{2}+2(n-1) \alpha q\right)}-\frac{\left(Q_{2}+2(n-1) \alpha q\right)}{2 q}\right]^{2} .
$$

For the case $\mathrm{g}=0$ we have

$$
R\left(a_{n}\right)=\left[\frac{\left(Q_{2}+2(n-1) \alpha q\right)}{2 q}\right]^{2}-\left[\frac{\left(Q_{2}+2 n \alpha q\right)}{2 q}\right]^{2}=\alpha^{2}(1-2 n)-\alpha \frac{Q_{2}}{q},
$$

which gives

$$
R\left(a_{n}\right)-R\left(a_{n-1}\right)=-2 \alpha^{2}
$$

then by using Eq. (40) we have

$$
R\left(a_{0}\right)=\alpha\left(\alpha-\frac{Q_{2}}{q}\right)
$$

We define the following operators

$$
K_{0}=\frac{1}{2 \alpha^{2}} R\left(a_{0}\right), \quad K_{ \pm}=\frac{1}{\alpha} B_{ \pm},
$$

Using Eqs. (41), (43) one can show that the shape invariant algebra for the five-parameter exponentialtype potential model is $s u(1,1)$

$$
\left[K_{+}, K_{-}\right]=-2 K_{0}, \quad\left[K_{0}, K_{-}\right]=-K_{-}, \quad\left[K_{0}, K_{+}\right]=K_{+}
$$

\section{Discussion}


By choosing appropriate parameters in the five-parameter model exponential-type potential one can construct seven typical potential $[18,19,20,21]$

\section{Case:1. $V_{0} \tanh ^{2}\left(\frac{r}{d}\right)$ potential}

Choosing $q=1, \alpha=\frac{1}{d}, \mathrm{~g}=0, Q_{3}=0$, and set

$$
Q_{2}^{2}-2 \alpha Q_{2}=4 V_{0}
$$

then the five-parameter exponential-type potential becomes the $V_{0} \tanh ^{2}\left(\frac{r}{d}\right)$ potential with constant shift.

$$
V(r)=V_{0} \tanh ^{2}\left(\frac{r}{d}\right)-V_{0}
$$

\section{Case:2. Scarf II potential}

If $q=1, \mathrm{~g}=0$, and put

$$
\begin{aligned}
Q_{2}^{2}-Q_{3}^{2}-2 \alpha Q_{2} & =-4\left[B^{2}-A(A+\alpha)\right], \\
Q_{2} Q_{3}+\alpha Q_{3} & =-2 B(2 A+\alpha),
\end{aligned}
$$

the potential (35) turns to the Scarf II potential

$$
V(r)=\left[B^{2}-A(A+\alpha)\right] \operatorname{sech}^{2} \alpha r+B(2 A+\alpha) \operatorname{sech} \alpha r \tanh \alpha r,
$$

\section{Case:3. Generalized Pöschl-Teller}

Choosing $q=-1, \mathrm{~g}=0$, and setting

$$
\begin{aligned}
Q_{2}^{2}+Q_{3}^{2}+2 \alpha Q_{2} & =4\left[B^{2}+A(A+\alpha)\right], \\
Q_{2} Q_{3}+\alpha Q_{3} & =-2 B(2 A+\alpha),
\end{aligned}
$$

the five-parameter exponential-type potential becomes the generalized pöschl-Teller potential

$$
V(r)=\left[B^{2}+A(A+\alpha)\right] \operatorname{cosech}^{2} \alpha r-B(2 A+\alpha) \operatorname{cosech} \alpha r \operatorname{coth} \alpha r,
$$

\section{Case:4. Pöschl-Teller II potential}


If we choose $q=-1, \mathrm{~g}=0, \alpha \rightarrow 2 \alpha$ and put

$$
\begin{gathered}
Q_{2}^{2}+Q_{3}^{2}+4 \alpha Q_{2}=8[A(A+\alpha)+B(B-\alpha)], \\
Q_{2} Q_{3}+2 \alpha Q_{3}=-4[A(A+\alpha)-B(B-\alpha)],
\end{gathered}
$$

the five-parameter exponential-type potential turns to the pöschl-Teller II potential potential

$$
V(r)=-A(A+\alpha) \operatorname{sech}^{2} \alpha r+B(B-\alpha) \operatorname{cosech}^{2} \alpha r
$$

\section{Case:5. PT-symmetric version of the Scarf II. potential}

Choosing $q=1, \mathrm{~g}=0$, and letting

$$
\begin{gathered}
Q_{2}^{2}-Q_{3}^{2}-2 \alpha Q_{2}=4 V_{1} \\
Q_{2} Q_{3}-\alpha Q_{3}=2 i V_{2}
\end{gathered}
$$

the five-parameter exponential-type potential yields the PT-symmetric version of the Scarf II potential

$$
V(r)=-V_{1} \operatorname{sech}^{2} \alpha r-i V_{2} \operatorname{sech} \alpha r \tanh \alpha r
$$

\section{Case:6. Symmetrical double-well potential}

If we take $q=1, \mathrm{~g}=0, Q_{3}=0$ and set

$$
Q_{2}^{2}-2 \alpha Q_{2}=4\left(V_{1}+V_{2}\right)
$$

Eq. (35) reduce to the standard symmetrical double-well potential with constant shift.[22]

$$
V(r)=V_{1} \tanh ^{2} \alpha r-\frac{V_{2}}{\cosh ^{2} \alpha r}-V_{1}
$$

\section{Case:7. Reflectionless-type potential}

If we define $q=1, \alpha=1, \mathrm{~g}=0, Q_{3}=0$ and put

$$
Q_{2}^{2}-2 \alpha Q_{2}=2 \lambda(\lambda+1)
$$

Eq. (35) turns to the reflectionless-type potential

$$
V(r)=-\frac{1}{2} \lambda(\lambda+1) \operatorname{sech}^{2} r
$$




\subsection{Harmonic Oscillator}

We take the scalar and vector potentials as follows:

$$
V(r)=\frac{V_{0}}{2} r^{2}
$$

Inserting these potentials into Eq. (34) we obtain

$$
\left\{-\frac{d^{2}}{d r^{2}}+(M+E) V_{0} r^{2}\right\} u(r)=\left(E^{2}-M^{2}\right) u(r) .
$$

The superpotential is

$$
W\left(r, a_{n}\right)=a_{n} r
$$

where

$$
a_{n}^{2}=a_{1}^{2}=(M+E) V_{0} .
$$

The remainder in Eq. (8) is given by

$$
R\left(a_{n}\right)=2 a_{n}=2 \sqrt{(M+E) V_{0}}
$$

Introducing the following operators

$$
K_{0}=\frac{R\left(a_{0}\right)}{\sqrt{(M+E) V_{0}}}=I, \quad K_{ \pm}=\frac{1}{\sqrt{(M+E) V_{0}}} B_{ \pm}
$$

so, we have

$$
\left[K_{-}, K_{+}\right]=I, \quad\left[K_{0}, K_{ \pm}\right]=0, \quad\left[K_{ \pm}, K_{+} K_{-}\right]=\mp K_{ \pm},
$$

where these commutation relations denote the Heisenberg-Wely algebra.

\subsection{Morse potential}

We consider scalar and vector exponential-type potentials written, respectively, as follows:

$$
S(r)=S_{0} e^{-\alpha r}, \quad V(r)=V_{0} e^{-\alpha r},
$$

substituting these potentials into Eq. (33) we have

$$
\left\{-\frac{d^{2}}{d r^{2}}+\left(S_{0}^{2}-V_{0}^{2}\right) e^{-2 \alpha r}+2\left(M S_{0}+E V_{0}\right) e^{-\alpha r}\right\} u(r)=\left(E^{2}-M^{2}\right) u(r) .
$$

which is called Klein-Gordon-Morse potential [3]. The superpotential is

$$
W\left(r, a_{n}\right)=a_{n}-\sqrt{S_{0}^{2}-V_{0}^{2}} e^{-\alpha r}
$$

where

$$
a_{n}=a_{1}-(n-1) \alpha, \quad a_{1}=-\frac{\alpha}{2}+\frac{M S_{0}+E V_{0}}{\sqrt{S_{0}^{2}-V_{0}^{2}}}
$$


The remainder is given by

$$
R\left(a_{n}\right)=\left(2 a_{n}-\alpha\right) \alpha
$$

We introduce the following operators

$$
K_{0}=\frac{R\left(a_{0}\right)}{2 \alpha^{2}}, \quad K_{ \pm}=\frac{B_{ \pm}}{\alpha}
$$

where

$$
R\left(a_{0}\right)=\frac{2\left(M S_{0}+E V_{0}\right)}{\sqrt{S_{0}^{2}-V_{0}^{2}}} .
$$

From Eq. (75) one can obtain that the shape invariant algebra for morse potential in Klein-Gordon equation to be $s u(1,1)$ algebra

$$
\left[K_{+}, K_{-}\right]=-2 K_{0}, \quad\left[K_{0}, K_{ \pm}\right]= \pm K_{ \pm}
$$

\section{Conclusion}

In this paper, we have briefly recalled the concepts of shape invariance method and its algebra structure. The shape invariant algebras, in general, have infinite-dimensional and under conditions they become finite-dimensional. We have considered the three-dimensional s-wave relativistic Klein-Gordon equation with equal scalar and vector five-parameter exponential-type potential. This equation satisfied the Shape invariant condition, then we showed a large class of exponential-type potentials can be obtain by choosing the appropriate parameters in this potential. By using the shape invariant algebra we have shown that the Lie algebra of five-parameter exponential-type potentials is $s u(1,1)$. Then we have considered the Harmonic oscillator potential and we found that this potential has the Heisenberg-Wely algebra. In the same process, we determined the Lie algebra structure of Morse potential with $s u(1,1)$ algebra in the Klein-Gordon equation.

\section{References}

[1] W. Greiner, Relativistic Quantum Mechanics, third ed., Springer, Berlin, 2000.

[2] T. Jana, P. Roy, Phys. Lett. A 361 (2007) 55.

[3] G. Chen, Z. Chen, P. Xuan, Phys. Lett. A 352 (2006)317.

G. Chen, Phys. Lett. A 339 (2005) 300.

[4] A. S. de Castro, Phys. Lett. A 338 (2005) 81.

[5] A. B. Balantekin, arXiv:quant-ph/9712018v1.

[6] E. Witten, Nucl. Phys. B 188 (1981) 513. 
[7] F. Cooper, B. Freeman, Ann. Phys. 146 (1983) 262.

F. Cooper, A. Khare, U. Sukhatme, Supersymmetry in Quantum Mechanics, World Scientific, Singapore, 2001.

[8] L. Gendenshtein, JETP Lett. 38 (1983) 356.

[9] A. Gangopadhyaya, U. Sukhatme, Phys. Lett. A 224 (1996) 5.

[10] E. D. Filho, R. M. Ricotta, J. Phys. A 37 (2004) 1057.

[11] T. Fukui, N. Aizawa, Phys. Lett. A 180, (1993), 308.

[12] A. B. Balantekin, Phys. Rev. A 57 (1998) 4188

[13] C. Chuan, J. Phys. A: Math. Gen. 24 (1991) L 1165

[14] A. B. Balantekin, M. A. Cândido Ribeiro and A. N. F. Aleixo, J. Phys. A: Math. Gen. 32 (1999) 2785

[15] A. N. F. Aleixo, A. B. Balantekin and M. A. Cândido Ribeiro, J. Phys. A: Math. Gen. 35 (2002) 9063

[16] M. M. Nieto, L. M. Simmons, Jr., Am. J. Phys. 47, 634 (1979)

[17] C. S. Jia, X. L. Zeng, L. T. Sun, Phys. Lett. A 294 (2002) 185.

[18] Y. F. Diao, L. Zh. Yi, C. S. Jia, Phys. Lett. A 332 (2004) 157.

[19] X. Q. Zhao, C. S. Jia, Q. B. Yang Phys. Lett. A 337 (2005) 189.

[20] C. S. Jia, Y. Li, Y. Sun, J. Y. Liu, L. T. Sun, Phys. Lett. A 311 (2003) 115.

[21] Parthasarathi, D. Parashar and R. S. Kaushal, J. Phys. A, 37 (2004) 781.

[22] F. Büyükkilic, H. Eğrifes, D. Hemirhan, Theor. Chem. Accounts 98 (1997) 192. 